\title{
Randomized Placebo-Controlled Trial Evaluating the Ophthalmic Safety of Single-Dose Tafenoquine in Healthy Volunteers
}

\author{
Jessica Ackert ${ }^{1} \cdot$ Khadeeja Mohamed $^{2}$. Jason S. Slakter ${ }^{3}$. Sherif El-Harazi ${ }^{4} \cdot$ Alessandro Berni $^{5} \cdot$ Hakop Gevorkyan $^{6}$. \\ Elizabeth Hardaker ${ }^{7}$. Azra Hussaini ${ }^{8}$. Siôn W. Jones ${ }^{7}$. Gavin C. K. W. Koh ${ }^{5}$. Jyoti Patel ${ }^{9}$. Scott Rasmussen ${ }^{10}$. \\ Deborah S. Kelly ${ }^{11}$. David E. Barañano ${ }^{12}$. John T. Thompson ${ }^{12} \cdot$ Keith A. Warren $^{10} \cdot$ Robert C. Sergott $^{13}$. \\ John Tonkyn ${ }^{14} \cdot$ Allen Wolstenholme $^{15} \cdot$ Hanna Coleman $^{3} \cdot$ Alex Yuan $^{3} \cdot$ Stephan Duparc $^{16} \cdot$ Justin A. Green $^{5}$
}

Published online: 11 June 2019

(c) The Author(s) 2019

\begin{abstract}
Introduction Tafenoquine has been recently registered for the prevention of relapse in Plasmodium vivax malaria. Objective This study assessed the pharmacodynamic effects of 300-mg single-dose tafenoquine on the retina.

Methods This phase I, prospective, multicenter, randomized, single-masked, placebo-controlled, parallel-group study was conducted between 2 February 2016 and 14 September 2017 at three US study centers. Adult healthy volunteers were randomized (2:1) to receive either a single 300-mg oral dose of tafenoquine or matched placebo on day 1. Ophthalmic assessments, including spectral domain optical coherence tomography (SD-OCT) and fundus autofluorescence (FAF), were conducted at baseline and day 90 and evaluated for pre-determined endpoints by an independent, masked reading center.

Results One subject in each group met the composite primary endpoint for retinal changes identified with SD-OCT or FAF, i.e., one out of $306(0.3 \%)$ with tafenoquine, one out of $161(0.6 \%)$ with placebo. Both cases had unilateral focal ellipsoid zone disruption at day 90 with no effect on best-corrected visual acuity. The tafenoquine-treated subject had this abnormality at baseline, and was enrolled in error. There was no difference in ophthalmic safety between tafenoquine and placebo.

Conclusion There was no evidence of any pharmacodynamic effect of 300-mg single-dose tafenoquine on the retina or any short-term clinically relevant effects on ophthalmic safety. This clinical trial is registered with ClinicalTrials.gov (identifier: NCT02658435).
\end{abstract}

\section{Key Points}

Tafenoquine is approved for Plasmodium vivax malaria relapse prevention.

In this placebo-controlled study in healthy volunteers, single-dose tafenoquine $300 \mathrm{mg}$ had no pharmacodynamic effect on the retina or clinically relevant effects on ophthalmic safety.

These findings are reassuring given that tafenoquine will be used in resource-poor areas, where access to ophthalmological care is often limited.

Electronic supplementary material The online version of this article (https://doi.org/10.1007/s40264-019-00839-w) contains supplementary material, which is available to authorized users.

Justin A. Green

justin.a.green@gsk.com

Extended author information available on the last page of the article

\section{Introduction}

Tafenoquine is an antimalarial drug for the prevention of relapse in Plasmodium vivax malaria-termed radical cure [1-3]. More than a third of the world's population are at risk of $P$. vivax malaria and it is the most prevalent form of malaria outside Africa [4]. Relapses are caused by reactivation of a dormant parasite liver state-the hypnozoite [5]. Patients can suffer multiple relapses recurring weeks or months after first becoming infected. Relapses also generate gametocytes, the sexual phase of the parasite, allowing onward human-to-mosquito transmission and maintaining the parasite in the population [5]. As hypnozoites are undetectable by any diagnostic method and cause no symptoms, infected individuals can silently harbor the parasite for extended periods. In this manner, the parasite evades malaria control efforts and can be transported to areas where malaria has been previously eliminated [6]. Thus, radical cure of $P$. vivax malaria, which kills both the blood-stage parasites that cause clinical disease and 
the hypnozoites that propagate relapse, is necessary to both reduce the burden of disease and accelerate malaria elimination [6]. Tafenoquine was recently approved by the United States Food and Drug Administration and the Australian Therapeutic Goods Administration, with approval from these stringent authorities supporting regulatory submissions in $P$. vivax-endemic countries to enable patient access achieved through their National Malaria Control Programs.

The 8-aminoquinolines primaquine and tafenoquine are the only available treatments that can clear $P$. vivax hypnozoites from the liver. These drugs must be given with a schizonticide to clear blood-stage parasites, usually 3 days of chloroquine. The recommended regimen for primaquine lasts 14 days $(15 \mathrm{mg} / \mathrm{kg} / \mathrm{day})$, and outside clinical trials, adherence is poor and effectiveness compromised [7]. In contrast, tafenoquine is given as a single 300-mg dose [1-3], a significant improvement in convenience, which should promote real-world clinical effectiveness. Chloroquine, which has known effects on the retina [8], is a 4-aminoquinoline, and although tafenoquine is an 8-aminoquinoline, as these two compounds will be coadministered, any theoretical risk of retinal adverse events with tafenoquine requires investigation.

Tafenoquine has been studied in malaria prophylaxis and treatment as well as $P$. vivax radical cure. Ophthalmic findings were reported in a study conducted in Australian soldiers randomized 3:1 to receive either a loading dose of $200 \mathrm{mg}$ daily tafenoquine for 3 days followed by weekly tafenoquine prophylaxis $(200 \mathrm{mg}$ ) or a loading dose of $250 \mathrm{mg}$ mefloquine/day for 3 days followed by weekly mefloquine prophylaxis $(250 \mathrm{mg})$ for 6 months [9]. Mild vortex keratopathy, which did not affect visual acuity, was observed in 69 out of 74 tafenoquine subjects (93\%) versus none out of 21 with mefloquine, and was fully resolved in all subjects by 1 year [9]. Retinal abnormalities were reported in $39.1 \%$ of tafenoquine subjects (27/69) and $23.5 \%$ (4/17) with mefloquine [9]. However, an expert ophthalmology review board convened to examine the data could not determine the relevance of the retinal findings because no baseline retinal photography data were available [9]. The board also noted that the retinal findings may have been normal variations [9]. Consequently, a followup ocular safety study was conducted comparing highdose tafenoquine monotherapy ( $400 \mathrm{mg}$ for 3 days) versus chloroquine (1500 $\mathrm{mg}$ for 3 days) followed by primaquine (15 mg for 14 days) in P. vivax malaria patients [10]. In this study, baseline data were available, and mild postbaseline keratopathy was observed in $31.8 \%$ of patients (14/44) with tafenoquine versus $0 \%(0 / 24)$ with chloroquine/primaquine. On clinical examination, mild postbaseline retinal findings were reported in ten out of 44 patients $(22.7 \%)$ receiving tafenoquine and two out of 24
(8.3\%) receiving chloroquine/primaquine $(P=0.15$; treatment difference $14.4 \%, 95 \%$ confidence interval $[\mathrm{CI}]-5.7$ to 30.8 ), but with no clinically relevant changes in visual acuity or macular function tests [10]. Masked digital photograph review by an independent reviewer indicated no changes from baseline at day 28 for either group [10]. At day 90 , one tafenoquine patient had a slight increase in atrophy versus baseline and one chloroquine/primaquine patient had retinal atrophy at baseline and a slight increase in atrophy versus baseline at day 90 , with no effect on visual acuity in either case [10].

In the development program for tafenoquine plus chloroquine in $P$. vivax radical cure, a single 300-mg tafenoquine dose was used, a therapy with a lower dose and shorter duration than that used in the studies outlined above that were conducted in prophylaxis or monotherapy treatment. Across three randomized clinical trials with tafenoquine $300 \mathrm{mg}$ plus chloroquine in $P$. vivax radical cure, ophthalmic findings were uncommon based on clinical examination: keratopathy was noted in $0.9 \%$ (1/107) of patients, retinal hyperpigmentation in $1.0 \%$ (1/102), and retinal hypopigmentation in 1.0\% (1/102) [2]. However, in these studies, retinal changes were assessed using clinical examination and masked reading of digital fundus photography. These methods are not consistent with recent recommendations [8], and have inadequate sensitivity to detect potential subtle changes in retinal health. Additional confounders included co-administration of chloroquine, which has known ophthalmic safety effects [8], and the disease under study, as symptomatic $P$. vivax malaria can also include ocular manifestations [11].

The current study assessed the pharmacodynamic effects of a 300-mg single oral dose of tafenoquine on the retina in comparison to placebo using spectral domain optical coherence tomography (SD-OCT) and fundus autofluorescence (FAF) in healthy volunteers using procedures consistent with American Academy of Ophthalmology guidelines [8]. Secondary objectives were to assess the overall ophthalmic safety of tafenoquine and general safety. The study is registered at ClinicalTrials.gov (identifier: NCT02658435).

\section{Methods}

\subsection{Study Design}

This was a phase I, prospective, multicenter, randomized, single-masked, placebo-controlled, parallel-group study of a $300-\mathrm{mg}$ single oral dose of tafenoquine in adult healthy volunteers. The study was conducted between 2 February 2016 and 14 September 2017 at three study centers in the USA. A central reading center (Digital Angiography 
Reading Center, Great Neck, NY, USA) masked to treatment allocation conducted the evaluation of SD-OCT, FAF and fundus photography outcomes. The study was conducted in accordance with the Declaration of Helsinki and all applicable local laws and followed Good Clinical Practice procedures. An independent review board at each participating center reviewed and approved the study protocol. All subjects provided written informed consent.

\subsection{Population}

Eligible participants were of either sex, aged $\geq 18$ to $\leq 45$ years, weighed $\geq 35$ to $\leq 100 \mathrm{~kg}$, and were healthy as determined by the investigator, with hematology and chemistry values within the normal range, and capable of giving signed informed consent. Full inclusion and exclusion criteria are listed in detail in Electronic Supplementary Material 1. Key general exclusion criteria were as follows: current or chronic history of liver disease, or known hepatic or biliary abnormalities, hemoglobin values outside the lower limit of normal range, phenotypic glucose-6-phosphate dehydrogenase (G6PD) deficiency (determined by a quantitative assay of enzyme activity; defined as $<70 \%$ of locally defined median value), and a Fredericia-corrected QTc interval of $>450 \mathrm{~ms}$. Subjects had to be of non-reproductive potential or capable of adhering to contraceptive requirements. Pregnant and lactating females were excluded. Key ophthalmic exclusion criteria were bilateral best-corrected visual acuity (BCVA) of $\leq 72$ letters, eye disease that could compromise ophthalmic assessments, intraocular surgery or laser photocoagulation within 3 months of dosing, high myopia (equal to, or worse than, - 6.00 diopters), anterior, intermediate or posterior uveitis or history of significant intraocular infectious disease or another active inflammatory disease, SD-OCT central subfield thickness $<250 \mu \mathrm{m}$ or $>290 \mu \mathrm{m}$, presence of significant abnormal patterns on FAF or ocular abnormalities on fundus photography at screening, or uncontrolled intraocular pressure $>22 \mathrm{mmHg}$.

\subsection{Randomization and Masking}

Eligible subjects were randomized in a 2:1 ratio to receive either a single 300-mg oral dose of tafenoquine (GlaxoSmithKline, Harlow, United Kingdom) or matched placebo on day 1 of the study (Fig. 1). Treatment was directly observed, administered with $240 \mathrm{~mL}$ of water and given with food. The randomization schedule was generated by GlaxoSmithKline Quantitative Sciences using validated internal software. The GlaxoSmithKline online randomization system (RAMOS NG) was used to allocate subjects to treatment. Subjects were masked to treatment; as were the readers at the independent retinal reading center, who reviewed the data. Although not required by the protocol, investigators were also masked to treatment allocation, with only pharmacy staff aware of treatment allocation throughout the study.

\subsection{Assessments}

Subjects were followed up for 90 days, with safety follow-up on day 7 ( \pm 1 day) and telephone contact for determination of adverse events and concomitant medication on days 30 and 60 ( \pm 7 days) (Fig. 1). Ophthalmic assessments were

Fig. 1 Study schematic

\begin{tabular}{|c|c|c|}
\hline Study phase & Study visit & Procedures \\
\hline \multirow[t]{2}{*}{ Screening (up to 30 days) } & Day -30 & $\begin{array}{l}\text { Screening assessments (inclusion/exclusion } \\
\text { assessments, ECG, laboratory tests) }\end{array}$ \\
\hline & Day -7 to 1 & $\begin{array}{l}\text { Baseline ophthalmic assessments (SD-OCT and } \\
\text { FAF, ophthalmic examination, BCVA and fundus } \\
\text { photography) }\end{array}$ \\
\hline Randomization and dosing & Day 1 & Tafenoquine $300 \mathrm{mg}$ or placebo $(2: 1)$ \\
\hline \multirow[t]{4}{*}{ Follow up } & Day 7 & $\begin{array}{l}\text { Safety follow up (adverse events, vital signs } \\
\text { and laboratory tests) }\end{array}$ \\
\hline & Day 30 & $\begin{array}{l}\text { Telephone contact: } \\
\text { adverse events/concomitant medications }\end{array}$ \\
\hline & Day 60 & $\begin{array}{l}\text { Telephone contact: } \\
\text { adverse events/concomitant medications }\end{array}$ \\
\hline & Day 90 & $\begin{array}{l}\text { Primary endpoint: retinal changes on SD-OCT } \\
\text { and FAF } \\
\text { Secondary endpoints: ophthalmic assessments } \\
\text { (SD-OCT and FAF, ophthalmic examination, BCVA } \\
\text { and fundus photography) and safety (adverse } \\
\text { events, ECGs and laboratory tests) }\end{array}$ \\
\hline
\end{tabular}

BCVA, best corrected visual acuity; ECG, electrocardiogram; FAF, fundus autofluorescence; SD-OCT, spectral domain optical coherence tomography. 
conducted at baseline (day -7 to day 1 pre-dose) and at day 90 ( -7 to +14 days) (Fig. 1).

A complete eye exam was conducted, i.e., pupil, motility and confrontation visual field examination; slit lamp evaluation of anterior segment structures (including cornea); and intraocular pressure measurement and dilated fundus examination (indirect ophthalmoscopy and slit lamp biomicroscopy, including the lens). BCVA was measured at a distance of $4 \mathrm{~m}$ using Early Treatment Diabetic Retinopathy Study (ETDRS) visual acuity charts.

SD-OCT, FAF and color fundus images were obtained by an appropriately trained technician using a protocol provided by the central reading center using approved equipment (SDOCT and FAF: SPECTRALIS ${ }^{\circledR}$ system, Heidelberg Engineering, Inc., Franklin, MA, USA; fundus photography: Zeiss FF4, Carl Zeiss Meditec, Dublin, CA, USA). Images were evaluated by the investigator and confirmed by the reading center for protocol inclusion/exclusion criteria and safety monitoring. For SD-OCT and FAF, pharmacodynamic effect was evaluated by the central reading center using protocol-defined criteria (see Electronic Supplementary Material 2 for details).

Planned safety evaluations were conducted at screening and days 7, 30, 60 and 90 (Fig. 1). Adverse events were recorded and categorized using MedDRA (version 20) preferred terms.

Triplicate 12-lead electrocardiograms (ECGs) were performed at screening, and standard clinical laboratory tests were performed at screening and day 7 (clinical chemistry, urinalysis and hematology, including methemoglobin).

\subsection{Objectives and Endpoints}

The primary objective of the study was to assess the pharmacodynamic effects of a 300-mg single oral dose of tafenoquine on the retina via SD-OCT and FAF in healthy adult volunteers. Secondary objectives were to assess the overall ophthalmic safety of tafenoquine and general safety.

The primary endpoint was the proportion of subjects in the tafenoquine group with significant protocol-defined retinal changes at day 90 compared with baseline. A subject was considered to have met the primary endpoint if any one of five parameters evaluated using SD-OCT or FAF indicated a change from baseline in either eye: (1) central subfield thickness change from baseline $\geq 40 \mu \mathrm{m}$ (automated reading); (2) total macular volume change of $\geq 10 \%$ from baseline (automated reading); (3) central retinal/lesion thickness change from baseline $\geq 40 \mu \mathrm{m}$ (manual reading); (4) ellipsoid zone disruption (manual reading); or (5) if there was any abnormal FAF pattern at follow-up (see Electronic Supplementary Material 2 for details). If the automated readings of central subfield thickness or total macular volume at either visit were missing or deemed unreliable by the independent masked reading center, then corresponding manual readings of the central retinal area in the same eye were used for the primary endpoint.

Secondary endpoints for ophthalmic safety were (a) the mean change from baseline at day 90 in key SD-OCT parameters, including central retinal/lesion thickness, central subfield thickness, total macular volume, central retinal/ lesion thickness, and ellipsoid zone disruption width; (b) the proportion of subjects with abnormal changes at day 90 versus baseline observed on FAF; (c) the mean change at day 90 versus baseline in BCVA; and (d) the proportion of subjects with vortex keratopathy from slit lamp examination. Changes for quantitative measures were defined as a change from baseline by at least $10 \%$ or by 2 standard deviations (SDs) from historical mean values for normal subjects. For qualitative assessments, new findings or worsening of findings from baseline exams considered to be of clinical importance by the masked reading center were reported as a change from baseline.

For general safety, secondary endpoints were the frequency of adverse events and serious adverse events, the severity of adverse events, and abnormal values or clinically important changes in laboratory tests. A serious adverse event was defined as one that resulted in death, was lifethreatening, required hospitalization, caused disability/incapacity, led to a congenital abnormality or birth defect, or was deemed serious by the investigator. Additionally, liver injury and impaired liver function with alanine transaminase $\geq 3$ times the upper limit of normal (ULN) plus either total bilirubin $\geq 2$ times the ULN or an international normalized ratio (INR) $>1.5$ was defined as a serious adverse event. A severe adverse event was defined as an event that prevents normal everyday activities as assessed by the investigator or a change in laboratory values graded for severity using the Common Terminology Criteria for Adverse Events (CTCAE) (version 4.03).

\subsection{Statistical Analysis}

The safety population included all subjects who received at least one dose of study medication. The primary endpoint was evaluated in the ophthalmic safety population, in which all subjects had valid baseline and day 90 SD-OCT and FAF measurements within the visit window that allowed derivation of the primary outcome. For the primary endpoint, the point estimate and the corresponding 95\% CI were calculated for the proportion of subjects with significant protocol-defined retinal changes from baseline to day 90. As a secondary endpoint, the difference in the proportion of subjects with clinically significant changes between tafenoquine and placebo was estimated. Sensitivity analyses for these endpoints were performed on the per-protocol analysis that excluded subjects with major protocol violations, and 
separately on an expanded ophthalmic safety population, including subjects with follow-up beyond the day $90+14$ day assessment window. A missing equals failure analysis of the primary endpoint was also conducted using the safety population.

For quantitative secondary ophthalmic endpoints, the change from baseline was summarized for the safety population. Adverse events, ECGs, vital signs and laboratory parameters were evaluated in the safety population and summarized descriptively.

There were no formal hypotheses tested in this study. A sample size of 300 subjects was planned to be treated with tafenoquine based on a $95 \%$ probability of detecting an event when the underlying risk of clinically significant retinal findings at 90 days follow-up was $1 \%$. A placebo control group of 150 subjects was used to compare the ophthalmic assessments between the two groups. To allow for $10 \%$ lost to follow-up, a total sample size of up to 500 subjects was planned to be enrolled in the study. With a smaller sample size of 275 subjects treated with tafenoquine, there was a
95\% probability of detecting an event if the underlying risk was considered to be $1.1 \%$.

\section{Results}

\subsection{Patient Disposition}

Of the 1522 subjects screened, 500 subjects were enrolled and randomized, of which 498 were treated and 486 (98\%) completed the study (Fig. 2). Two subjects randomized to tafenoquine did not receive study medication and were not included in any analyses. Baseline characteristics were balanced between the two groups (Table 1). The ophthalmic safety population included 306 out of 330 subjects (92.7\%) in the tafenoquine group and 161 out of $168(95.8 \%)$ in the placebo group (Fig. 2). Major protocol deviations were observed in 21 subjects in the ophthalmic safety population, which led to their exclusion from the per-protocol population (Fig. 2).

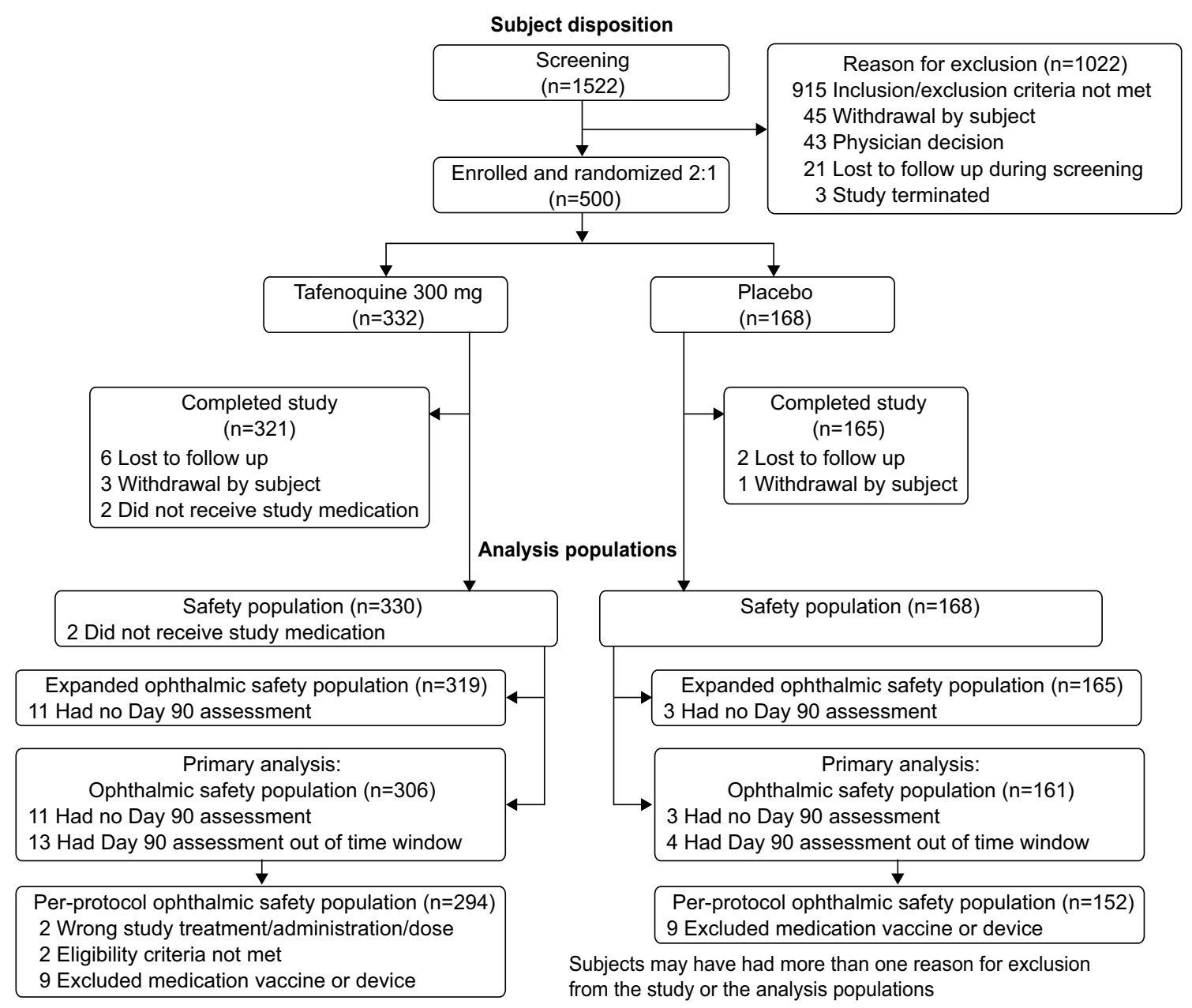

Fig. 2 Subject disposition 
Table 1 Baseline characteristics

\begin{tabular}{lll}
\hline Characteristic & Placebo $(n=168)$ & Tafenoquine $(n=330)$ \\
\hline Female sex, $n(\%)$ & $98(58.3)$ & $194(58.8)$ \\
Mean age, years (SD) [range] & $30.0(7.2)[17-45]$ & $29.0(7.2)[17-45]$ \\
Ethnicity, $n(\%)$ & & \\
White/Caucasian/European & $92(54.8)$ & $187(56.7)$ \\
African American/African & $58(34.5)$ & $114(34.5)$ \\
Asian & $7(4.2)$ & $16(4.8)$ \\
American Indian/Alaskan native & $1(0.6)$ & $2(0.6)$ \\
Native Hawaiian/Pacific Islander & $3(1.8)$ & 0 \\
Multiple & $7(4.2)$ & $11(3.3)$ \\
Mean G6PD enzyme activity, IU/g Hb (SD) & $10.5(1.2)[7.5-15.3]$ & $10.7(1.2)[7.6-16.1]$ \\
[range] & &
\end{tabular}

$G 6 P D$ glucose-6-phosphate dehydrogenase, $H b$ hemoglobin, $S D$ standard deviation

Fig. 3 Spectral domain optical coherence tomography (SD-OCT) images in the two subjects meeting the primary endpoint for retinal changes who received a 300-mg singledose tafenoquine (note that the subject had this abnormality at baseline and was enrolled in error) or $\mathbf{b}$ placebo

\section{(a) Tafenoquine-treated subject}

\section{Baseline}

(i)

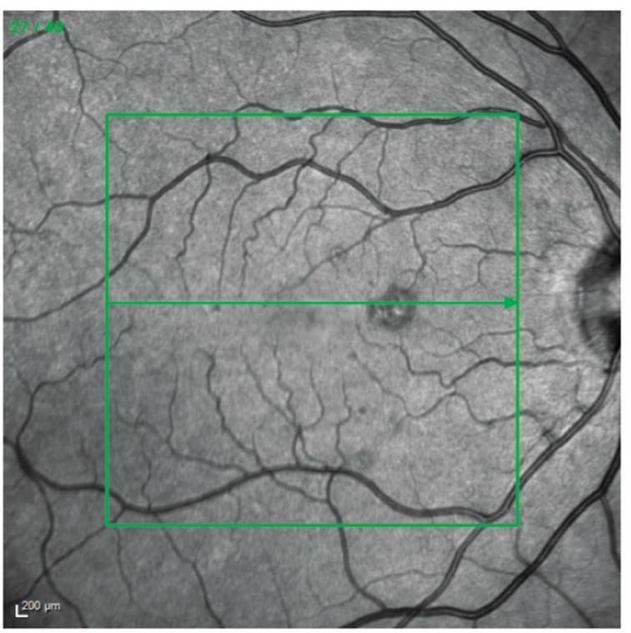

(iii)

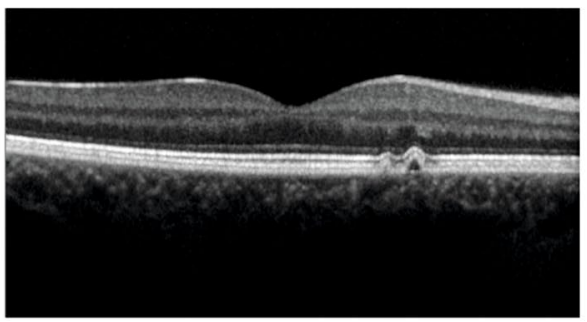

Day 90

(ii)

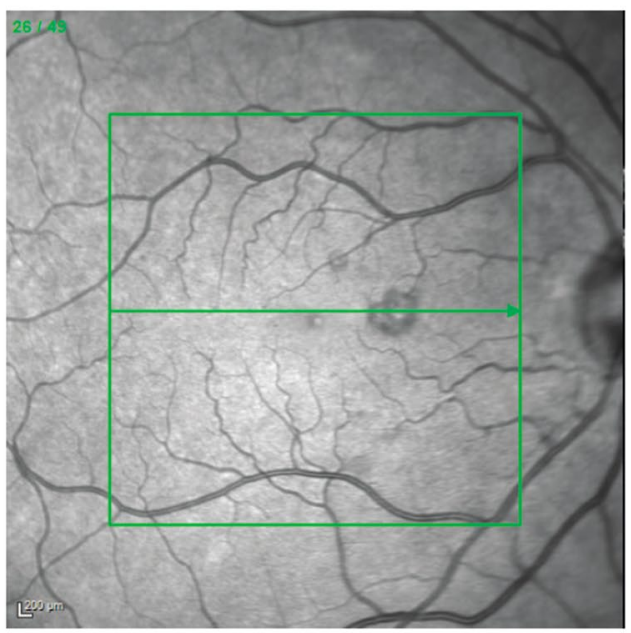

(iv)

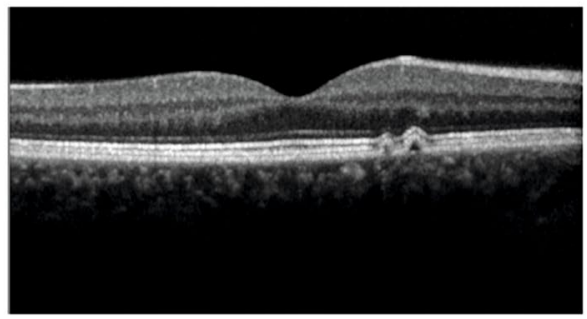

At baseline, small areas of retinal pigmentary change were noted in the infrared en face OCT image (i) which was unchanged at Day 90 (ii). The green horizontal line with arrow notes the anatomical location of the corresponding SD-OCT scan. The baseline SD-OCT image showed two small pigment epithelial detachments with overlying ellipsoid zone disruption (iii) which remained unchanged at Day 90 (iv).

\subsection{Ophthalmic Safety}

\subsubsection{Primary Outcome: Retinal Changes from Baseline}

One subject in each group met the primary endpoint for retinal changes identified with SD-OCT or FAF, i.e., one out of $306(0.3 \%)$ with tafenoquine and one out of 161 $(0.6 \%)$ with placebo (Fig. 3) (see Electronic Supplementary Material 3). Both cases had unilateral focal ellipsoid zone disruption, with no changes in either eye for the other four parameters in the primary endpoint and no visual adverse events or changes in BCVA. The upper limit of the $95 \% \mathrm{CI}$ 
Fig. 3 (continued)

(b) Placebo-treated subject

\section{Baseline}

(i)

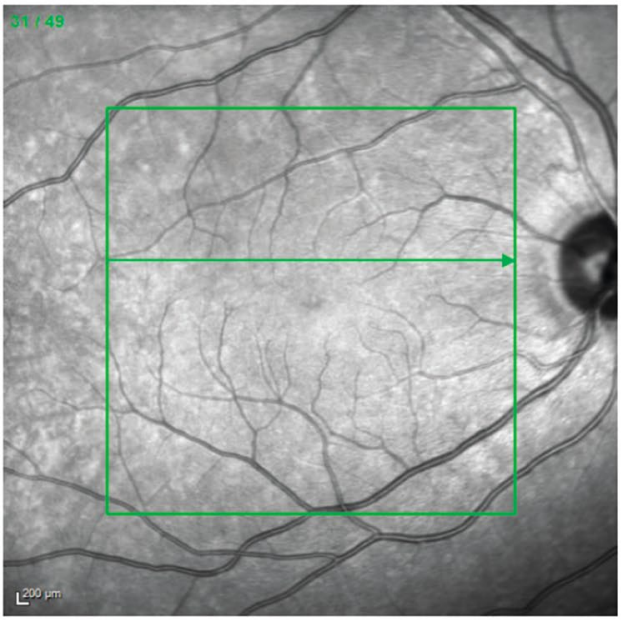

(iii)

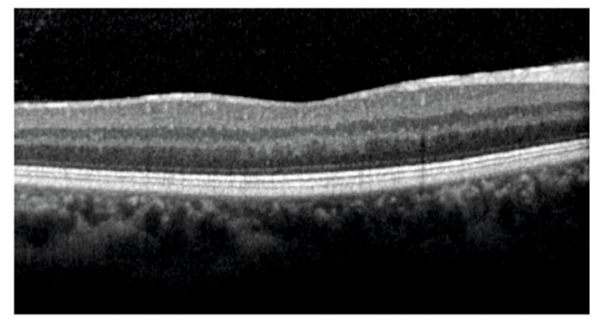

Day 90

(ii)

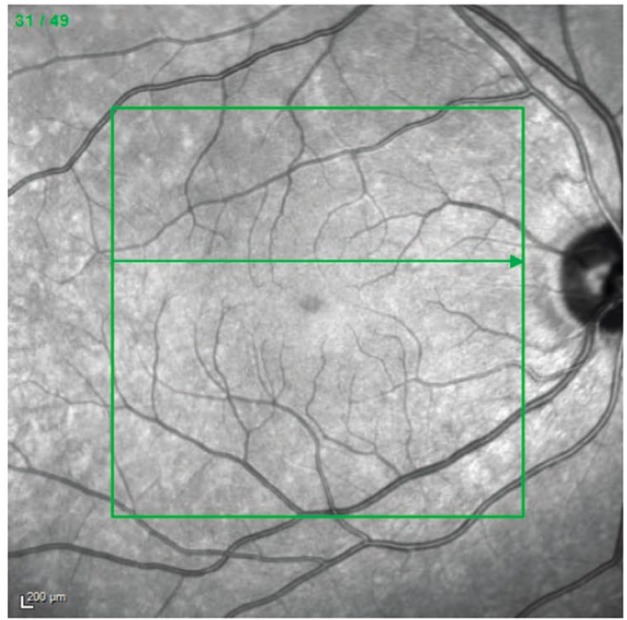

(iv)

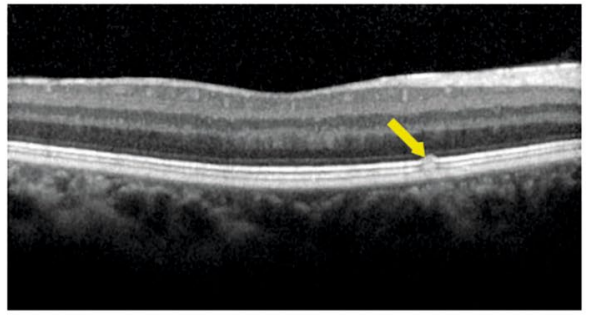

This subject had a unilateral ellipsoid zone disruption of $>15 \%$ representing a change from baseline in the right eye (iv, yellow arrow represents abnormality). The subject did not have ellipsoid zone disruption at baseline (iii). The green horizontal line with arrow on the infrared images ( $i$ and ii) notes the anatomical location of the corresponding SD-OCT scans.

for the proportion of subjects with retinal changes in either eye with tafenoquine was $1.5 \%$, and the treatment difference between tafenoquine and placebo was $-0.3 \%$ (95\% CI -3.1 to 1.3 ). There were no subjects with bilateral retinal changes in the study. The upper limit of the $95 \%$ CI for the proportion of subjects with retinal changes in both eyes following tafenoquine $(0 / 306)$ was $0.9 \%$, with a treatment difference of $0 \%(95 \% \mathrm{CI}-2.3 \%, 1.2 \%)$. The sensitivity analysis which included all subjects with day 90 data irrespective of the assessment window did not identify any further subjects with protocol-defined retinal changes.

The tafenoquine-treated subject with focal ellipsoid zone disruption at day 90 also had this finding at baseline and was, therefore, enrolled into the study in error and was excluded from the per-protocol population. The focal disruption was related to small retinal pigment epithelial detachments. Thus, in the per-protocol analysis, none of 294 subjects ( $0 \%$; upper limit for $95 \%$ CI $0.9 \%$ ) had retinal changes with tafenoquine and one of $152(0.7 \%)$ had such changes with placebo; treatment difference $-0.7 \%$ (95\% CI $-3.6 \%$, $0.7 \%$ ). For the subject in the placebo group with ellipsoid zone disruption during the study, there was no detectable or known cause for this disruption.

One tafenoquine subject had a missing central subfield thickness measurement at baseline. Seven other subjects (four placebo; three tafenoquine) had large changes from baseline recorded in central subfield thickness as a result of misalignment of images between baseline and day 90 visits using the automated software algorithm. In these eight cases, for the primary endpoint, the results were imputed from the categorical change from baseline measured manually in central retinal/lesion thickness for the same eye.

\subsubsection{Secondary Ophthalmic Safety Outcomes}

Quantitative results of SD-OCT parameters did not show any treatment differences in central subfield thickness, central retinal/lesion thickness, macular cube volume, or subretinal fluid thickness (Table 2; see also Electronic Supplementary Material 4 and 5). For intraocular pressure, there was no clinically important change from baseline to day 90 or any difference between treatment arms (Electronic 
Table 2 Treatment comparison for tafenoquine versus placebo of the change from baseline to day 90 in quantitative optical confocal tomography results (safety population)

\begin{tabular}{|c|c|c|c|c|c|c|c|}
\hline \multirow[t]{2}{*}{ Outcome } & \multirow[t]{2}{*}{ Change } & \multirow[t]{2}{*}{ Eye } & \multicolumn{2}{|c|}{$N$ subjects } & \multicolumn{2}{|c|}{ Mean value } & \multirow{2}{*}{$\begin{array}{l}\text { Treatment difference } \\
(95 \% \mathrm{CI})\end{array}$} \\
\hline & & & $\begin{array}{l}\text { Placebo } \\
(n=168)\end{array}$ & $\begin{array}{l}\text { Tafenoquine } \\
(n=330)\end{array}$ & $\begin{array}{l}\text { Placebo } \\
(n=168)\end{array}$ & $\begin{array}{l}\text { Tafenoquine } \\
(n=330)\end{array}$ & \\
\hline \multirow{6}{*}{$\begin{array}{l}\text { Central subfield thick- } \\
\text { ness }(\mu \mathrm{m})^{*}\end{array}$} & \multirow[t]{3}{*}{ Decrease } & Right & 74 & 119 & -3.1 & -3.0 & $0.0(-0.6$ to 0.7$)$ \\
\hline & & Left & 62 & 125 & -3.5 & -3.0 & $0.4(-0.5$ to 1.3$)$ \\
\hline & & Selected & 94 & 176 & -3.6 & -3.4 & $0.2(-0.5$ to 0.9$)$ \\
\hline & \multirow[t]{3}{*}{ Increase } & Right & 72 & 149 & 4.4 & 3.2 & $-1.2(-2.6$ to 0.2$)$ \\
\hline & & Left & 79 & 141 & 3.7 & 3.9 & $0.1(-0.9$ to 1.2$)$ \\
\hline & & Selected & 99 & 203 & 4.8 & 3.9 & $-0.8(-2.1$ to 0.4$)$ \\
\hline \multirow{6}{*}{$\begin{array}{l}\text { Central retinal/lesion } \\
\text { thickness }(\mu \mathrm{m})\end{array}$} & \multirow[t]{3}{*}{ Decrease } & Right & 47 & 100 & -4.2 & -4.2 & $0.0(-1.3$ to 1.2$)$ \\
\hline & & Left & 51 & 96 & -4.3 & -4.0 & $0.4(-0.8$ to 1.5$)$ \\
\hline & & Selected & 75 & 147 & -4.8 & -4.5 & $0.3(-0.8$ to 1.3$)$ \\
\hline & \multirow[t]{3}{*}{ Increase } & Right & 111 & 199 & 6.7 & 6.7 & $0.0(-1.3$ to 1.2$)$ \\
\hline & & Left & 105 & 202 & 7.7 & 6.7 & $-1.0(-2.4$ to 0.3$)$ \\
\hline & & Selected & 135 & 254 & 8.2 & 7.7 & $-0.4(-1.7$ to 0.8$)$ \\
\hline \multirow{6}{*}{$\begin{array}{l}\text { Macular cube volume } \\
(\mu \mathrm{m})^{*}\end{array}$} & \multirow[t]{3}{*}{ Decrease } & Right & 71 & 127 & -0.05 & -0.06 & $-0.01(-0.03$ to 0.00$)$ \\
\hline & & Left & 66 & 124 & -0.05 & -0.05 & $0.00(-0.01$ to 0.01$)$ \\
\hline & & Selected & 92 & 170 & -0.06 & -0.07 & $-0.01(-0.02$ to 0.00$)$ \\
\hline & \multirow[t]{3}{*}{ Increase } & Right & 79 & 160 & 0.06 & 0.06 & 0.00 ( -0.02 to 0.02$)$ \\
\hline & & Left & 85 & 167 & 0.06 & 0.07 & 0.00 (0.00 to 0.02$)$ \\
\hline & & Selected & 111 & 206 & 0.07 & 0.07 & $0.00(-0.01$ to 0.02$)$ \\
\hline
\end{tabular}

The selected eye is the one with the larger magnitude change by direction

Only endpoints with an increase or decrease are included

Only one subject in each group had a change in ellipsoid zone disruption from baseline to day 90 (see Fig. 3). There were no subjects with any subretinal fluid thickness at baseline or day 90

CI confidence interval

*Inner limiting membrane-retinal pigment epithelium. Excludes the results of eight subjects where change from baseline central subfield thickness was imputed from change from baseline central retinal/lesion thickness for the primary endpoint

Supplementary Material 6). Vortex keratopathy was present in one subject in the placebo group at baseline, but resolved by day 90 . New-onset vortex keratopathy at day 90 was recorded in both eyes for one subject in the tafenoquine group (1/307; upper limit of 95\% CI 1.4\%), but not in the placebo group (0/162; treatment difference $0.3 \%$ [95\% CI -0.2 to 1.8$]$ ). However, post-database lock, it was discovered that, because of a data entry error, the case of vortex keratopathy in the tafenoquine group had in fact been noted by the ophthalmologist as a LASIK scar with calcium deposits, present at baseline and day 90 . This subject did not, therefore, have vortex keratopathy, but the results have been reported as recorded in the study.

\subsubsection{Best-Corrected Visual Acuity}

Absolute values at day 90 and change from baseline to day 90 for BCVA did not indicate any clinically meaningful effect of tafenoquine (Table 3). There was no treatment difference for BCVA (logMAR) between tafenoquine and placebo $(0.012$
[95\% CI -0.002 to 0.027]; i.e., < 1 letter on the Snellen scale), and the categorical results of BCVA were also similar between the two groups (see Electronic Supplementary Material 7 and 8). Scatter plots of SD-OCT parameters versus BCVA were produced to explore any association of structural and functional changes (Fig. 4). No patterns indicating any effect of tafenoquine were identified from these analyses.

\subsection{General Safety}

The frequency of adverse events of any cause was similar between the tafenoquine $(26.1 \%$ [86/330]) and placebo (25.0\% [42/168]) groups (Table 4; see also Electronic Supplementary Material 9). Headache, nausea and vomiting were more common with tafenoquine than placebo (Table 4). There were no deaths and no serious or severe adverse events during the study, nor any adverse events leading to study withdrawal. All adverse events in the tafenoquine group were mild in severity. In the placebo group, two adverse events were of moderate severity (tooth abscess and 
Table 3 Best-corrected visual acuity at baseline and day 90 and change from baseline to day 90

\begin{tabular}{|c|c|c|c|c|c|}
\hline \multicolumn{6}{|l|}{ Absolute values } \\
\hline Study group & Eye & Visit & $N$ & Mean (SD) [range], logMAR & Snellen ratio* [range] \\
\hline \multirow[t]{4}{*}{ Placebo $(n=168)$} & \multirow[t]{2}{*}{ Right } & Baseline & 168 & $-0.055(0.087)[-0.2$ to 0.2$]$ & $20 / 16[20 / 12.5$ to $20 / 32]$ \\
\hline & & Day 90 & 162 & $-0.056(0.096)[-0.3$ to 0.4$]$ & $20 / 16[20 / 10$ to $20 / 50]$ \\
\hline & \multirow[t]{2}{*}{ Left } & Baseline & 168 & $-0.045(0.092)[-0.3$ to 0.2$]$ & $20 / 20[20 / 10$ to $20 / 32]$ \\
\hline & & Day 90 & 162 & $-0.044(0.100)[-0.3$ to 0.4$]$ & $20 / 20[20 / 10$ to $20 / 50]$ \\
\hline \multirow[t]{4}{*}{ Tafenoquine $(n=330)$} & \multirow[t]{2}{*}{ Right } & Baseline & 330 & $-0.048(0.094)[-0.2$ to 0.3$]$ & $20 / 20[20 / 12.5$ to $20 / 40]$ \\
\hline & & Day 90 & 308 & $-0.043(0.095)[-0.3$ to 0.3$]$ & $20 / 20[20 / 10$ to $20 / 40]$ \\
\hline & \multirow[t]{2}{*}{ Left } & Baseline & 330 & $-0.041(0.095)[-0.3$ to 0.3$]$ & $20 / 20[20 / 10$ to $20 / 40]$ \\
\hline & & Day 90 & 308 & $-0.029(0.098)[-0.3$ to 0.4$]$ & $20 / 20[20 / 10$ to $20 / 50]$ \\
\hline \multicolumn{6}{|c|}{ Change from baseline to day 90} \\
\hline Study group & Eye & Visit & $N$ & Mean (SD) [range] to logMAR & $\begin{array}{l}\text { Mean (SD) [range], } \\
\text { number of letters }\end{array}$ \\
\hline \multirow[t]{2}{*}{ Placebo $(n=168)$} & Right & Day 90 & 162 & $-0.004(0.089)[-0.2$ to 0.3$]$ & $0(3.41)[-11$ to 11$]$ \\
\hline & Left & Day 90 & 162 & $0.001(0.082)[-0.2$ to 0.2$]$ & $0.2(3.58)[-10$ to 12$]$ \\
\hline \multirow[t]{2}{*}{ Tafenoquine $(n=330)$} & Right & Day 90 & 308 & $0.005(0.088)[-0.2$ to 0.3$]$ & $-0.3(3.35)[-12$ to 10$]$ \\
\hline & Left & Day 90 & 308 & $0.011(0.090)[-0.3$ to 0.4$]$ & $-0.4(3.4)[-11$ to 13$]$ \\
\hline
\end{tabular}

SD standard deviation

*Snellen visual acuity ratio equivalent to mean and range logMAR visual acuity

limb injury). One pregnancy was reported in the tafenoquine group ( 7 weeks pregnant at the day 90 assessment), the outcome of the pregnancy was unknown as the patient was lost to follow-up, despite multiple attempts at contact.

The incidence of drug-related adverse events was higher with tafenoquine $(9.7 \%$ [32/330]) than with placebo $(2.4 \%$ [4/168]). This was mainly because of a higher rate of nausea $(3.6 \%$ [12/330] vs $0.6 \%$ [1/168]) in subjects receiving tafenoquine (see Electronic Supplementary Material 10). There was no difference between treatment groups in the incidence of ophthalmic adverse events (Table 5). ECGs were only performed at baseline, with no clinically relevant findings (data not shown).

Clinical chemistry values and changes from baseline showed no values or patterns of concern and were consistent with the expected tafenoquine safety profile (see Electronic Supplementary Material 11 and 12) [12]. There was no clinically important effect of tafenoquine on hematological parameters, except for methemoglobin, which was mildly increased from baseline to day 7 with tafenoquine (mean change $0.41 \%$ [SD 0.58]) compared with placebo (0.06\% [SD 0.40]) (see Electronic Supplementary Material 13 and 14).

\section{Discussion}

This placebo-controlled study reports the pharmacodynamic effect of 300-mg single-dose tafenoquine in healthy subjects followed up for 90 days. Retinal changes from baseline were assessed using SD-OCT and FAF using procedures consistent with guidelines from the American Academy of Ophthalmology [8]. Additionally, clinical examination and BCVA evaluation was conducted. The methods used had the required sensitivity to detect early retinal changes that might have resulted from tafenoquine treatment. Ophthalmic safety and general safety were secondary objectives.

One subject in the tafenoquine group met the primary endpoint of a retinal change from baseline, with ellipsoid zone disruption associated with small retinal pigment detachment seen on SD-OCT in one eye at day 90. However, re-examination of the subject's screening images clearly showed that he had this pre-existing abnormality, visible in the images, noted by the masked reading center, but not flagged as a screen fail. Therefore, this subject should have been excluded from the study, but was inadvertently enrolled. There were no other retinal or ophthalmic findings for this subject, and the observed abnormality was in a single eye. Thus, no tafenoquine subject met the primary endpoint in the per-protocol population. One subject in the placebo group had unilateral ellipsoid zone disruption that fulfilled the criteria for the primary endpoint.

Benign, reversible vortex keratopathy has been reported in previous tafenoquine studies following high doses and/or long-term therapy [9, 10]. In clinical trials with single-dose tafenoquine $300 \mathrm{mg}$ in $P$. vivax patients, there was one case out of 107 subjects $(0.9 \%)$ of unilateral vortex keratopathy, and this is included on the product labelling as an uncommon potential adverse reaction [12]. 


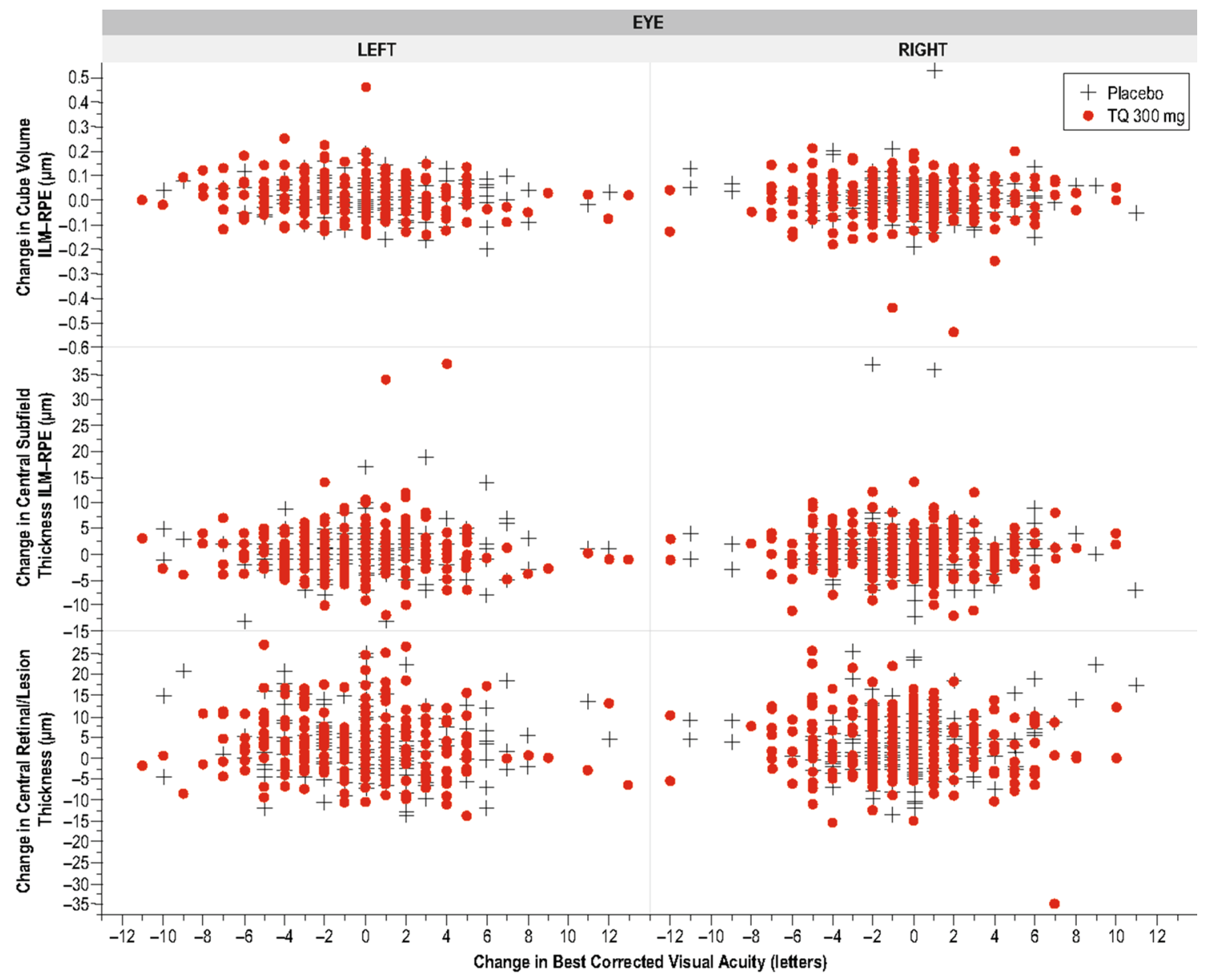

Fig. 4 Change in baseline for best-corrected visual acuity versus optical coherence tomography parameters. No subjects had subretinal fluid thickness. Excludes the central subfield thickness results of sub-

Table 4 Most common adverse events of any cause

\begin{tabular}{lll}
\hline Preferred term, $n(\%)$ & $\begin{array}{l}\text { Placebo } \\
(n=168)\end{array}$ & $\begin{array}{l}\text { Tafenoquine } \\
(n=330)\end{array}$ \\
\hline Any event & $42(25.0)$ & $86(26.1)$ \\
Headache & $9(5.4)$ & $23(7.0)$ \\
Nausea & $1(0.6)$ & $14(4.2)$ \\
Upper respiratory tract infection & $4(2.4)$ & $8(2.4)$ \\
Viral upper respiratory tract infection & $4(2.4)$ & $4(1.2)$ \\
Vomiting & $1(0.6)$ & $5(1.5)$ \\
Back pain & $2(1.2)$ & $3(0.9)$ \\
Diarrhea & $2(1.2)$ & $2(0.6)$ \\
Gastroenteritis & $2(1.2)$ & $2(0.6)$ \\
Toothache & $2(1.2)$ & $2(0.6)$ \\
Viral pharyngitis & $2(1.2)$ & $2(0.6)$ \\
Tension headache & $2(1.2)$ & 0
\end{tabular}

Adverse events occurring in $\geq 1 \%$ of subjects in either treatment group. For a full listing of adverse events see Electronic Supplementary Material 9 jects with imputed change from baseline and one tafenoquine (TQ) subject that had a missing baseline value (4 placebo; 4 TQ)

In this study, although vortex keratopathy was reported in one tafenoquine subject, the subject had a LASIK scar with calcium deposits that was observed at both visits which was inaccurately coded to keratopathy. Thus, there were no confirmed vortex keratopathy reported in this study.

No serious or severe adverse events were reported. There was a higher percentage of participants reporting nausea in the tafenoquine group; otherwise there were no clinically meaningful differences between the two treatment arms in the nature, frequency or severity of adverse events reported, including ophthalmic adverse events. There were no unexpected clinical laboratory abnormalities.

One subject in the tafenoquine arm reported mydriasis and photophobia, which were self-limiting and required no intervention. These adverse events were temporally associated with tafenoquine administration (on the tafenoquine dosing day and day 2). As similar events of this nature are recognized with other quinoline antimalarial 
Table 5 All ophthalmic adverse events of any cause

\begin{tabular}{lll}
\hline $\begin{array}{l}\text { System organ class/preferred term, } \\
(\%)\end{array}$ & $\begin{array}{l}\text { Placebo } \\
(n=168)\end{array}$ & $\begin{array}{l}\text { Tafenoquine } \\
(n=330)\end{array}$ \\
\hline $\begin{array}{l}\text { Infections and infestations } \\
\text { Conjunctivitis }\end{array}$ & 0 & $1(0.3)$ \\
Eye disorders & & \\
Any event & $7(4.2)$ & $9(2.7)$ \\
Conjunctivitis allergic & $1(0.6)$ & $1(0.3)$ \\
Eye irritation & 0 & $2(0.6)$ \\
Photophobia & $1(0.6)$ & $1(0.3)$ \\
Vision blurred & $1(0.6)$ & $1(0.3)$ \\
Astigmatism & $1(0.6)$ & 0 \\
Blepharospasm & $1(0.6)$ & 0 \\
Corneal deposits & 0 & $1(0.3)$ \\
Dry eye & 0 & $1(0.3)$ \\
Eye disorder & 0 & $1(0.3)$ \\
Foreign body sensation in eyes & 0 & $1(0.3)$ \\
Mydriasis & 0 & $1(0.3)$ \\
Presbyopia & $1(0.6)$ & 0 \\
Retinal exudates & 0 & $1(0.3)$ \\
Retinal hemorrhage & $1(0.6)$ & 0 \\
Hepatobiliary disorders & & $1(0.3)$ \\
Ocular icterus & 0 & \\
\hline
\end{tabular}

drugs, tafenoquine causality cannot be excluded, and consequently, tafenoquine product labelling includes photophobia as a potential adverse event [12].

The primary limitation of this study is that it was conducted in adult healthy individuals without pre-existing eye conditions and so does not reflect the patient population likely to receive tafenoquine. This was necessary to fully investigate the pharmacodynamic potential of tafenoquine to cause retinal effects in the absence of significant confounding factors. However, it does not exclude the possibility of an effect of tafenoquine on the retina in individuals with pre-existing retinal damage or in the presence of $P$. vivax malaria. Ophthalmic assessments using clinical examination and digital fundus photography were included in phase IIb and phase III studies in $P$. vivax radical cure, with mild retinal hypo/hyperpigmentation observed in two out of 102 patients $(2.0 \%)$ treated with tafenoquine and one out of $56(1.8 \%)$ treated with primaquine [1-3]. There are also potential methodological limitations, as although the methods used were sensitive to detect retinal changes, further investigations, such as microperimetry and multifocal electroretinography, were not included.

In conclusion, consistent with data from the phase IIb and phase III studies evaluating a single $300-\mathrm{mg}$ dose of tafenoquine for the radical cure of $P$. vivax malaria [1-3], there was no indication of any clinically important shortterm ocular risk from the use of 300-mg tafenoquine single-dose treatment in this placebo-controlled study in adult healthy volunteers. These findings are reassuring given that tafenoquine will be used in resource-poor areas, where access to ophthalmological care is often limited.

Acknowledgements Naomi Richardson of Magenta Communications Ltd wrote the first draft of this article from the clinical study report, collated author contributions, provided graphic and editorial services and was funded by GlaxoSmithKline. The authors acknowledge with thanks the subjects who volunteered to participate in the study and the research site staff.

Author contributions Conception and design of the work/project: Jessica Ackert, Jason S. Slakter, Deborah S. Kelly, Justin A. Green, Allen Wolstenholme, Robert C. Sergott, and Khadeeja Mohamed. Acquisition of data: Sherif El-Harazi, Hakop Gevorkyan, Azra Hussaini, Jyoti Patel, Scott Rasmussen, David E. Barañano, John T. Thompson, Keith A. Warren, Hanna Coleman, Alex Yuan, and Jason S. Slakter. Conceptualization of the manuscript and review and synthesis of the literature: Jessica Ackert and Justin A. Green. Analysis and interpretation of data: Khadeeja Mohamed, Jessica Ackert, Elizabeth Hardaker, Alessandro Berni, Siôn W. Jones, Gavin C.K.W Koh, Allen Wolstenholme, Stephan Duparc, Justin A. Green, John Tonkyn, and Robert C. Sergott. All authors critically reviewed the manuscript and approved its submission for publication.

\section{Compliance with Ethical Standards}

Funding This multicenter study was funded and managed by GlaxoSmithKline and Medicines for Malaria Venture (GSK study number: 201807) and is registered with ClinicalTrials.gov (identifier: NCT02658435).

Conflict of interest Jessica Ackert, Khadeeja Mohamed, Alessandro Berni, Elizabeth Hardaker, Siôn W. Jones, Gavin C.K.W Koh, Jyoti Patel, John Tonkyn, Allen Wolstenholme, and Justin A. Green are employees of GlaxoSmithKline and hold shares in the company. Jason S. Slakter, Hakop Gevorkyan, Hanna Coleman, and Alex Yuan are employees of DARC. Hakop Gevorkyan is an employee of California Clinical Trials Medical Group, and Azra Hussaini is an employee of PAREXEL (California Clinical Trials Medical Group in association with PAREXEL International were contracted and reimbursed for conducting this study). Sherif El-Harazi is an employee of the Lugene Eye Institute; Scott Rasmussen and Keith A. Warren are employees of IQVIA; Deborah S. Kelly is a former employee of GSK and a current employee of Spark Therapeutics; David E. Barañano, John T. Thompson, and Robert C. Sergott received research funding for participating in this study, but have no other relevant conflicts. Robert C. Sergott is an employee of the Wills Eye Hospital. Stephan Duparc is an employee of Medicines for Malaria Venture.

Ethical approval An independent review board at each participating center reviewed and approved the study protocol.

Patient consent All subjects provided written informed consent.

Data sharing statement Anonymized individual participant data on study documents can be requested for further research from http:// www.clincalstudydatarequest.com.

Open Access This article is distributed under the terms of the Creative Commons Attribution-NonCommercial 4.0 International License 
(http://creativecommons.org/licenses/by-nc/4.0/), which permits any noncommercial use, distribution, and reproduction in any medium, provided you give appropriate credit to the original author(s) and the source, provide a link to the Creative Commons license, and indicate if changes were made.

\section{References}

1. Lacerda M, Llanos-Cuentas A, Krudsood S, Lon C, Saunders D, Mohammed R, et al. Single-dose tafenoquine to prevent relapse of Plasmodium vivax malaria. N Engl J Med. 2018;380(3):215-28.

2. Llanos-Cuentas A, Lacerda M, Tinh Hien T, Vélez I, Namaik-larp $\mathrm{C}$, Chu $\mathrm{C}$, et al. Tafenoquine versus primaquine to prevent relapse of Plasmodium vivax malaria. N Engl J Med. 2018;380(3):229-41.

3. Llanos-Cuentas A, Lacerda MV, Rueangweerayut R, Krudsood S, Gupta SK, Kochar SK, et al. Tafenoquine plus chloroquine for the treatment and relapse prevention of Plasmodium vivax malaria (DETECTIVE): a multicentre, double-blind, randomised, phase 2b dose-selection study. Lancet. 2014;383(9922):1049-58.

4. World Health Organization. World Malaria Report. WHO, Geneva. 2018. https://www.who.int/malaria/publications/world -malaria-report-2018/report/en/. Accessed 22 Nov 2018.

5. Adams JH, Mueller I. The biology of Plasmodium vivax. Cold Spring Harb Perspect Med. 2017;7(9):a025585.

6. World Health Organization. Strategy for malaria elimination in the Greater Mekong Subregion (2015-2030). World Health Organization, Geneva. 2015. http://iris.wpro.who.int/bitstream/handl e/10665.1/10945/9789290617181_eng.pdf;jsessionid=3204C
3285C2E6290730A3453BFFD279D? sequence $=1$. Accessed 27 Feb 2019.

7. Galappaththy GN, Tharyan P, Kirubakaran R. Primaquine for preventing relapse in people with Plasmodium vivax malaria treated with chloroquine. Cochrane Database Syst Rev. 2013(10):CD004389.

8. Marmor MF, Kellner U, Lai TY, Melles RB, Mieler WF, American Academy of Ophthalmology. Recommendations on screening for chloroquine and hydroxychloroquine retinopathy (2016 revision). Ophthalmology. 2016;123(6):1386-94.

9. Nasveld PE, Edstein MD, Reid M, Brennan L, Harris IE, Kitchener SJ, et al. Randomized, double-blind study of the safety, tolerability, and efficacy of tafenoquine versus mefloquine for malaria prophylaxis in nonimmune subjects. Antimicrob Agents Chemother. 2010;54(2):792-8.

10. Warrasak S, Euswas A, Fukuda M, Ittiverakul M, Miller R, Krudsood S et al. Comparative ophthalmic assessment of patients receiving tafenoquine or chloroquine/primaquine in a randomized clinical trial for Plasmodium vivax malaria radical cure. Int Ophthalmol. 2018; (E-publication ahead of print). https://doi. org/10.1007/s10792-018-1003-2.

11. Kochar A, Kalra P, Sb V, Ukirade V, Chahar A, Kochar DK, et al. Retinopathy of vivax malaria in adults and its relation with severity parameters. Pathog Glob Health. 2016;110(4-5):185-93.

12. GlaxoSmithKline. KRINTAFEL (tafenoquine) tablets, for oral use. GSK, Research Triangle Park. 2018. https://www.acces sdata.fda.gov/drugsatfda_docs/label/2018/210795s000lbl.pdf. Accessed 28 Oct 2018.

\section{Affiliations}

\section{Jessica Ackert ${ }^{1} \cdot$ Khadeeja Mohamed $^{2}$. Jason S. Slakter ${ }^{3} \cdot$ Sherif El-Harazi $^{4} \cdot$ Alessandro Berni $^{5} \cdot$ Hakop Gevorkyan $^{6}$. Elizabeth Hardaker ${ }^{7}$. Azra Hussaini ${ }^{8}$. Siôn W. Jones ${ }^{7}$. Gavin C. K. W. Koh ${ }^{5}$. Jyoti Patel ${ }^{9}$. Scott Rasmussen ${ }^{10}$. Deborah S. Kelly ${ }^{11}$. David E. Barañano ${ }^{12}$. John T. Thompson ${ }^{12} \cdot$ Keith A. Warren $^{10} \cdot$ Robert C. Sergott $^{13}$. John Tonkyn ${ }^{14} \cdot$ Allen Wolstenholme $^{15} \cdot$ Hanna Coleman $^{3} \cdot$ Alex Yuan $^{3} \cdot$ Stephan Duparc $^{16} \cdot$ Justin A. Green $^{5}$}

1 Clinical Research and Development, GlaxoSmithKline, Collegeville, PA, USA

2 Quantitative Sciences, GlaxoSmithKline Research and Development, Stockley Park West, Uxbridge, Middlesex, UK

3 Digital Angiography Reading Center (DARC), Great Neck, New York, NY, USA

4 Lugene Eye Institute, Glendale, CA, USA

5 Global Health, GlaxoSmithKline Research and Development Ltd., Stockley Park West, Uxbridge, Middlesex UB11 1BT, UK

6 California Clinical Trials Medical Group in affiliation with Parexel International, Glendale, CA, USA

7 Safety Evaluation and Risk Management, GlaxoSmithKline Research and Development, Stockley Park West, Uxbridge, Middlesex, UK
8 PAREXEL, Harbor Hospital, Baltimore, Baltimore, MD, USA

9 Clinical Data Management, GlaxoSmithKline Research and Development, Collegeville, PA, USA

10 IQVIA, Overland Park, KS, USA

11 Global Clinical Safety and Pharmacovigilance, GlaxoSmithKline, Collegeville, PA, USA

12 Retina Specialists, Baltimore, MD, USA

13 Neuro-Ophthalmology Service, Wills Eye Hospital, Philadelphia, PA, USA

14 GSK Medicines Research Centre, GlaxoSmithKline, Stevenage, Hertfordshire, UK

15 GlaxoSmithKline, Upper Providence, Collegeville, PA, USA

16 Research and Development, Medicines for Malaria Venture, Geneva, Switzerland 